\title{
Anti-Inflammatory Role of Cilostazol in Vascular Smooth Muscle Cells In vitro and In vivo
}

\author{
Chie Aoki, Yoshiyuki Hattori , Atsuko Tomizawa, Teruo Jojima, and Kikuo Kasai
}

Department of Endocrinology and Metabolism, Dokkyo University School of Medicine, Tochigi, Japan

\begin{abstract}
Aim: Cilostazol is a selective inhibitor of phosphodiesterase 3, by which it increases intracellular cAMP and activates protein kinase $\mathrm{A}$, thereby inhibiting platelet aggregation and inducing peripheral vasodilation. We investigated whether cilostazol might prevent nuclear factor (NF)- $\kappa$ B activation by activating AMP-activated protein kinase (AMPK) in vascular smooth muscle cells (VSMC).

Methods and Results: Cilostazol was observed to activate AMPK, as well as its downstream target, acetyl-CoA carboxylase, in rat VSMC. Phosphorylation of AMPK with cilostazol was not affected by co-treatment with an adenylate cyclase inhibitor, SQ 22536. Furthermore, a cell-permeable cyclic AMP analog, pCTP-cAMP, did not influence cilostazol-induced AMPK phosphorylation. These findings suggest that cilostazol-induced AMPK activation occurs through a signalling pathway independent of cyclic AMP. Cilostazol dose-dependently inhibited LPS-induced NF- $\kappa$ B activation in the present study. It was also observed to inhibit LPS-induced iNOS gene promoter activity and iNOS gene expression, resulting in markedly reduced NO production. An AMPK inhibitor compound C or siRNA for AMPK attenuated the observed cilostazol-induced inhibition of NF- $\kappa$ B activation by LPS. Ingestion of cilostazol inhibited NF- $\kappa$ B activation, as well as the induction of iNOS mRNA and protein expression, within the aortas of LPS-treated rats.

Conclusion: In light of these findings, we suggest that cilostazol might attenuate cytokine-induced expression of the iNOS gene by inhibiting NF- $\kappa$ B following AMPK activation in VSMC.
\end{abstract}

J Atheroscler Thromb, 2010; 17:503-509.

Key words; Vascular smooth muscle, Inflammation, AMPK, NF- $\kappa$ B, cAMP

\section{Introduction}

Cilostazol is an antiplatelet agent that inhibits platelet aggregation induced by collagen, 5'-adenosine diphosphate (ADP), epinephrine, and arachidonic acid $^{1)}$. Since being approved in the United States for the treatment of intermittent claudication in 1999, cilostazol has shown promise in the treatment of a number of cardiovascular disorders. Unlike other antiplatelet agents, cilostazol not only inhibits platelet function, but also appears to have beneficial effects on endothelial cell function ${ }^{1}$. We recently demonstrated that cilostazol inhibits the cytokine-induced expres-

Address for correspondence: Yoshiyuki Hattori, Department of Endocrinology and Metabolism, Dokkyo University School of Medicine, Mibu, Tochigi 321-0293, Japan

E-mail: yhattori@dokkyomed.ac.jp

Received: July 25, 2009

Accepted for publication: November 5, 2009 sion of various pro-inflammatory and adhesion molecule genes by suppressing NF- $\kappa$ B activity via AMPactivated protein kinase (AMPK) activation, and not via the cyclic AMP (cAMP)/protein kinase A (PKA) pathway ${ }^{2,3)}$. This is the mechanism by which cilostazol likely protects endothelial cells from inflammation. To show that this mechanism applies in vivo, we examined the aortas of cilostazol-treated rats to see whether there might be a relationship between the protective effect of cilostazol and AMPK expression. We observed high levels of AMPK expression in the aortas of cilostazol-treated rats compared with control rats, and strong induction of AMPK expression in the aortic smooth muscle cell layer of cilostazol-treated rats. The present study was therefore performed to investigate whether cilostazol might exert an antiinflammatory effect on lipopolysaccharide (LPS)treated vascular smooth muscle cells (VSMC) in culture and within the aortic tissue of LPS-injected rats. 


\section{Methods}

\section{Cell Culture}

We digested thoracic aortas from male Wistar rats using elastase and collagenase, after which isolated VSMC $^{4)}$ were cultured in DMEM containing $10 \%$ heat-inactivated FBS, $100 \mu \mathrm{g} / \mathrm{mL}$ piperacillin, and $100 \mu \mathrm{g} / \mathrm{mL}$ streptomycin. The experiments were performed using VSMC after 8-12 passages.

We complied with the guidelines outlined in the Guide for the Care and Use of Laboratory Animals published by the US National Institute of Health, as well as with the Declaration of Helsinki.

\section{Western Blot Analysis}

VSMC treated with cilostazol for various intervals were lysed using cell lysis buffer (Cell Signaling, Beverly, MA, USA) with $1 \mathrm{mM}$ PMSF. The protein concentration of each sample was measured using a Bio-Rad detergent-compatible protein assay. Subsequently, $\beta$-mercaptoethanol was added to a final concentration of $1 \%$, after which each sample was denatured by boiling for $3 \mathrm{~min}$. Samples containing $10 \mathrm{mg}$ protein were resolved by electrophoresis on $12 \%$ sodium dodecyl sulphate (SDS)-polyacrylamide gel and transferred to a polyvinyldienefluoride (PVDF) membrane (Bio-Rad, Tokyo, Japan), after which the samples were incubated with anti-phospho-Thr-172 AMPK polyclonal antibody and anti-phospho-Ser-79 acetyl-CoA carboxylase (ACC) polyclonal antibody (1:1,000, Cell Signaling). The binding of each of these antibodies was detected using sheep anti-rabbit IgG horseradish peroxidase $(1: 20,000)$ and an ECL Plus system (Amersham, Buckinghamshire, UK).

\section{NF- $\kappa$ B Activation}

To study NF- $\kappa$ B activation, VSMC were stably transfected with a cis-reporter plasmid containing the luciferase reporter gene linked to five repeats of $\mathrm{NF}-\kappa \mathrm{B}$ binding sites (pNF $\kappa \mathrm{B}-\mathrm{Luc}$; Stratagene, La Jolla, CA, USA), as previously described ${ }^{5)}$. For this, the $\mathrm{pNF} \kappa \mathrm{B}-\mathrm{Luc}$ plasmid was transfected together with a pSV2neo helper plasmid (Clontech, Palo Alto, CA, USA) into rat VSMC using a FuGEN 6 transfection reagent (Boehringer Mannheim, Mannheim, Germany). The cells were then cultured in the presence of G418 (Clontech) at a concentration of $500 \mathrm{mg} / \mathrm{mL}$ and the medium was replaced every 2 to 3 days. Approximately three weeks after transfection, G418resistant clones were isolated using a cloning cylinder and analyzed individually for the expression of luciferase activity. Several clones were also selected for analysis of $\mathrm{NF}-\kappa \mathrm{B}$ activation. Luciferase activity was mea- sured using a luciferase assay kit (Stratagene).

We also measured changes in the levels of NF- $\kappa \mathrm{B}$ p50 and p65 in nuclear extracts from the aorta using a transcription factor assay kit (Active Motif Japan, Tokyo, Japan). Nuclear extracts were prepared with a NE-PER nuclear extraction reagent (Pierce, Rockford, IL, USA), after which p50 and p65 were quantified using Jurkat nuclear extract as the standard.

\section{siRNA Transfection}

The day before transfection, plates were inoculated with an appropriate number of VSMC in serumcontaining medium to ensure $50-70 \%$ confluence the following day. AMPK $\alpha 1$ siRNA (Santa Cruz Biotechnology, Santa Cruz, CA, USA) mixed with siLentFect (BioRAd, Tokyo Japan) was added to the cells at a concentration of $10 \mathrm{nmol} / \mathrm{L}$. Forty-eight hours after transfection, NF- $\kappa \mathrm{B}$ activity was measured and compared between cells transfected with control siRNA and AMPK $\alpha 1$ siRNA.

\section{Production of NO and iNOS Promoter Analysis}

We studied the iNOS promoter function as previously described ${ }^{6}$. In brief, we used rat VSMC stably transfected with a construct containing a $1.7-\mathrm{kb}$ fragment of the iNOS promoter cloned in front of a reporter gene encoding the secreted form of human placental alkaline phosphatase (SEAP). We measured SEAP levels within the cell culture medium using a sensitive chemiluminescent assay (Phospha-Light; TROPIX, Bedford, MA, USA). Nitrite accumulation, which is an indicator of NO synthesis, was measured in the culture medium of confluent VSMC ${ }^{6}$.

\section{Real-Time PCR of iNOS mRNA}

For quantitative measurement of mRNA, $2 \mu \mathrm{g}$ of total RNA was treated with DNase I for $15 \mathrm{~min}$ and subsequently used for cDNA synthesis. Reverse transcription was performed using a SuperScript preamplification system (Gibco BRL, Gaithersburg, MD) with random oligonucleotide primers. The following primers were used: iNOS forward 5'-CTGCAGGTCTTTGACGCTCGG-3' and reverse 5'-GTGGAACACAGGGGTGATGCT-3', as previously described ${ }^{7}$. A typical reaction $(50 \mu \mathrm{L})$ contained $1 / 50$ of reverse transcription (RT)-generated $\mathrm{cDNA}$ and $200 \mathrm{nM}$ primer in $1 \times$ SYBR Green RealTime Master Mix (Toyobo, Tokyo, Japan) buffer. PCR reactions were carried out in a LineGene system (BioFlux, Tokyo, Japan) under the following conditions: $95^{\circ} \mathrm{C}$ for 5 min, followed by 40 cycles at $95^{\circ} \mathrm{C}$ for $15 \mathrm{sec}, 60^{\circ} \mathrm{C}$ for $15 \mathrm{sec}$, and $72^{\circ} \mathrm{C}$ for $30 \mathrm{sec}$. 


\section{Animals and Treatment}

Twelve male Sprague-Dawley rats (12 weeks of age) were obtained from Tokyo Experimental Animals (Tokyo, Japan) and divided into two groups as follows: control group (rats fed regular chow for two weeks) and cilostazol group (SD rats fed regular chow containing $0.1 \%$ cilostazol for two weeks). Thoracic aortas from rats in each group were isolated for immunohistochemical analysis, and the remaining rats were injected with LPS $(5 \mathrm{mg} / \mathrm{kg}$, i.p:, Sigma, St Louis, MO, USA), after which iNOS mRNA expression within the aorta was examined. The cilostazol doses were based on those used in human and rat experiments to date. The present experiments were reviewed and approved by the Committee on Ethics of Animal Experiments. The present study complied with the guidelines outlined in the Guide for the Care and Use of Laboratory Animals published by the US National Institutes of Health (NIH Publication No. 85-23, revised 1996).

\section{Immunohistochemical Analysis of AMPK Expres- sion in the Aorta}

Paraffin sections of $2 \mathrm{~mm}$ were prepared for analysis using the avidin-biotin-horseradish peroxidase complex method. The sections were de-paraffinized and incubated for $15 \mathrm{~min}$ in $3 \% \mathrm{H}_{2} \mathrm{O}_{2}$ to block endogenous peroxidase activity. After treatment with blocking serum, the sections were incubated with 1:200 dilution of AMPK polyclonal antibody (Cell Signaling) for $60 \mathrm{~min}$. The sections were then rinsed with Tris-buffered saline with $0.1 \%$ Tween 20 and incubated with biotinylated secondary antibody against sheep immunoglobulin (Dako, Glostrup, Denmark) for $20 \mathrm{~min}$. After rinsing again with Tris-buffered saline with $0.1 \%$ Tween 20 , the sections were incubated with horseradish peroxidase-conjugated streptavidin for $20 \mathrm{~min}$, followed by incubation with the peroxidase substrate solution, diaminobenzidine. The sections were then counterstained with hematoxylin and examined under light microscopy.

\section{Statistical Analysis}

Data are presented as the mean \pm SEM. Multiple comparisons were evaluated by ANOVA followed by Fisher's protected least significant difference test. A value of $p<.05$ was considered significant.

\section{Results}

\section{Cilostazol Induces AMPK Protein Expression}

We performed an animal study to examine the effect of cilostazol on AMPK activation within the rat
A

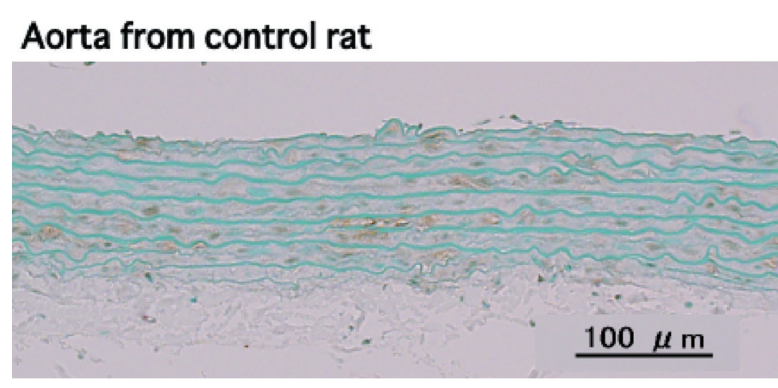

Aorta from cilostazol-treated rat

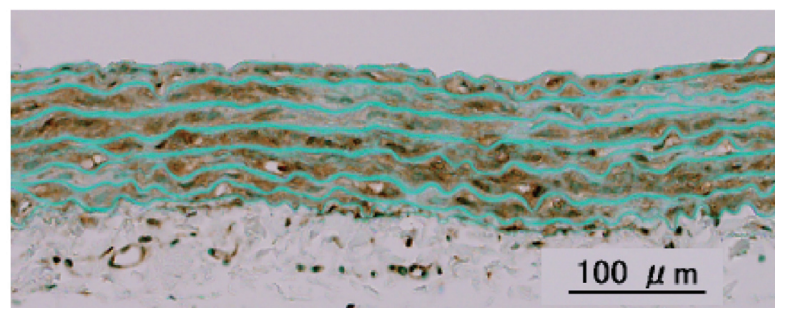

B

AMPK

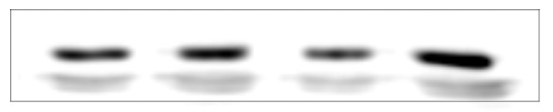

$\alpha-$ Tubulin

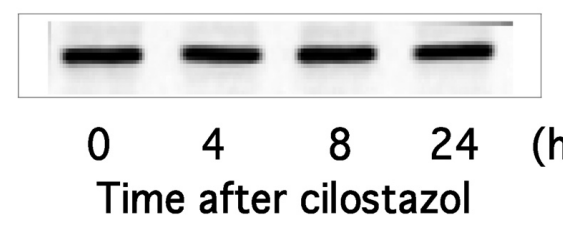

Fig. 1. (A) Immunohistochemical staining for AMPK in aortic tissue from rats fed cilostazol and cilostazol-free diets. Similar immunohistochemical profiles were observed in the aortic samples of 4 to 5 rats fed cilostazol and cilostazol-free diets. (B) Rat VSMC cells treated with cilostazol $(100 \mu \mathrm{M})$ for the indicated time periods before lysis, after which samples of cell lysate were probed with antibodies specific for AMPK and $\alpha$-tubulin.

aorta. As shown in Fig. 1A, marked AMPK staining was noted in the rat aorta following treatment with cilostazol for two weeks. Induction of AMPK expression was mainly observed within the aortic smooth muscle cell layer of cilostazol-treated rats. We also examined whether induction of AMPK protein expression might occur in cultured VSMC following treatment with cilostazol, and observed significant induction of AMPK protein expression in cilostazoltreated VSMC (Fig. 1B).

\section{Cilostazol Activates AMPK in VSMC Independently of cAMP}

Treatment of VSMC with cilostazol produced 
A

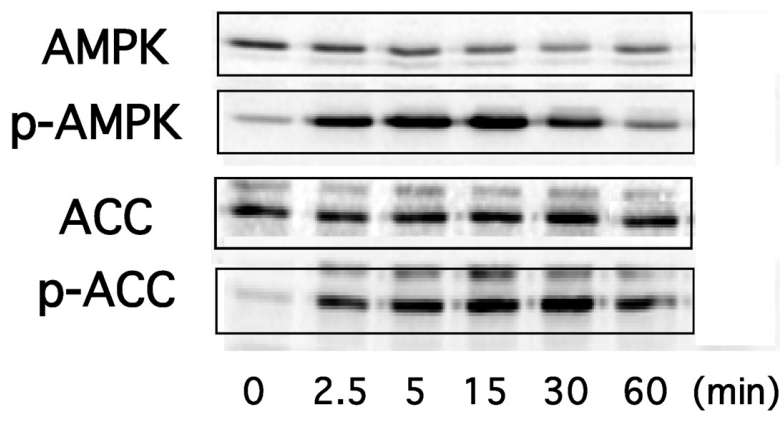

B

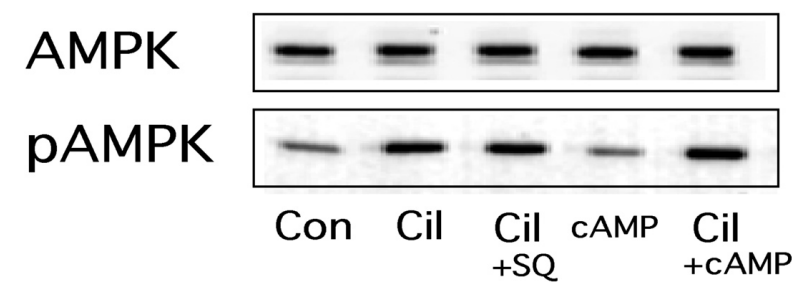

Fig. 2. (A) Cilostazol-mediated activation of $A M P K$ in rat VSMC. VSMC were treated with cilostazol $(100 \mu \mathrm{M})$ for the indicated time periods before lysis, after which samples of cell lysate were probed with antibodies specific for the phosphorylated forms of AMPK and acetyl-CoA carboxylase (ACC). (B) HUVEC treated with cilostazol $(100 \mu \mathrm{M})$ alone or in the presence of an adenylate cyclase inhibitor SQ $22536(10 \mu \mathrm{M})$ or a cell-permeable cAMP analog PCTP-cAMP $(100 \mu \mathrm{M})$. After $15 \mathrm{~min}$ of incubation, the cells were lysed and p-AMPK activity was analyzed. Three independent studies showed similar results.

time-dependent activation of AMPK, as suggested by the phosphorylation of AMPK, as well as its downstream target ACC (Fig. 2A). Phosphorylation of AMPK by cilostazol was not affected by co-treatment with an adenylate cyclase inhibitor SQ 22536 (Fig. 2B). A cell-permeable cAMP analog pCTPcAMP $(100 \mu \mathrm{M})$ did not induce AMPK phosphorylation, and had no effect on cilostazol-induced AMPK phosphorylation (Fig. 2B). Thus, cilostazol activates AMPK independently of cAMP in VSMC. We also examined whether other PDE3 inhibitors might activate AMPK in VSMC. Neither milrinone nor vesnarinone induced AMPK activation (data not shown).

\section{Cilostazol inhibits LPS-induced NF- $\kappa$ B activation in VSMC}

We initially examined whether different concentrations of cilostazol activated AMPK. Even $1 \mu \mathrm{M}$ cilostazol could phosphorylate AMPK and higher concentrations of cilostazol more evidently induced AMPK phophrylation (Fig. 3A). We then examined the effect of cilostazol on LPS-induced NF- $\kappa$ B activa-
A

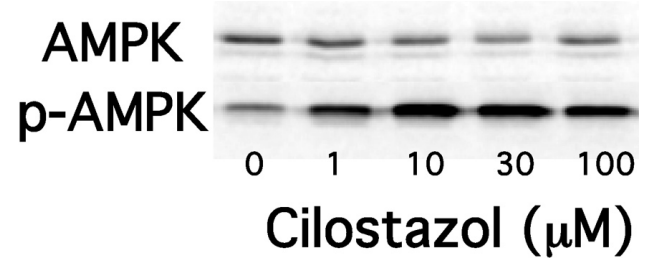

B
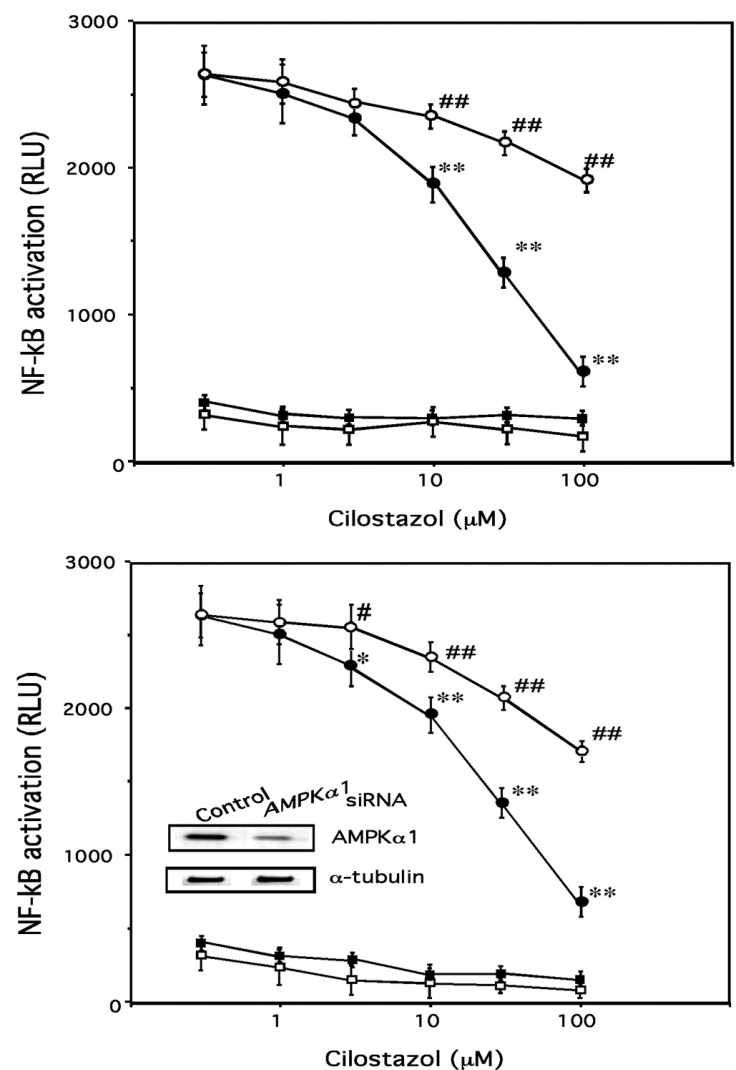

Fig. 3. (A) AMPK activation by cilostazol. Cells were treated with different concentrations of cilostazol for $15 \mathrm{~min}$ and AMPK phosphorylation was assessed by Western blotting analysis. (B) Cilostazol-mediated inhibition of LPS-induced NF- $\kappa$ B activation.

Upper Fig.: Cilostazol dose-dependently suppressed LPS-mediated activation of NF- $\kappa \mathrm{B}$-dependent transcriptional activity, which was significantly attenuated in cells treated with AMPK inhibitor compound $\mathrm{C}(\mathrm{CC}: 10 \mu \mathrm{M})$. Closed squares (control) and open squares (CC) represent the results obtained in the absence of LPS, while closed circles (control) and open circles (CC) represent the results obtained in the presence of LPS. Lower Fig.: Cilostazol dosedependently suppressed LPS-mediated activation of NF- $\kappa$ B-dependent transcriptional activity, which was significantly attenuated in cells transfected with siRNA for AMPK $\alpha 1$. Closed squares (control siRNA) and open squares (AMPK $\alpha 1$ siRNA) represent the results obtained in the absence of LPS, while closed circles (control siRNA) and open circles (AMPK $\alpha 1$ siRNA)) represent the results obtained in the presence of LPS Results represent mean values \pm SEM $(n=6) .{ }^{* *} p<0.01$ vs. NF- $\kappa \mathrm{B}$ activity in the absence of cilostazol, ${ }^{\#} p<0.05, \# p<0.01$ vs. NF- $\kappa$ B activity in control at the same concentration of cilostazol. 
A

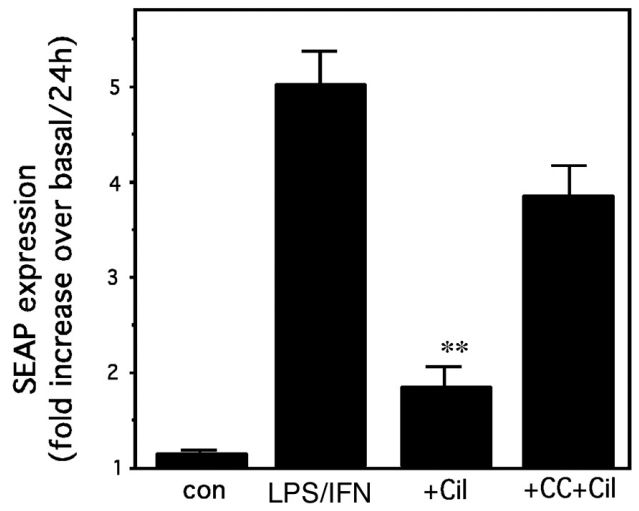

B

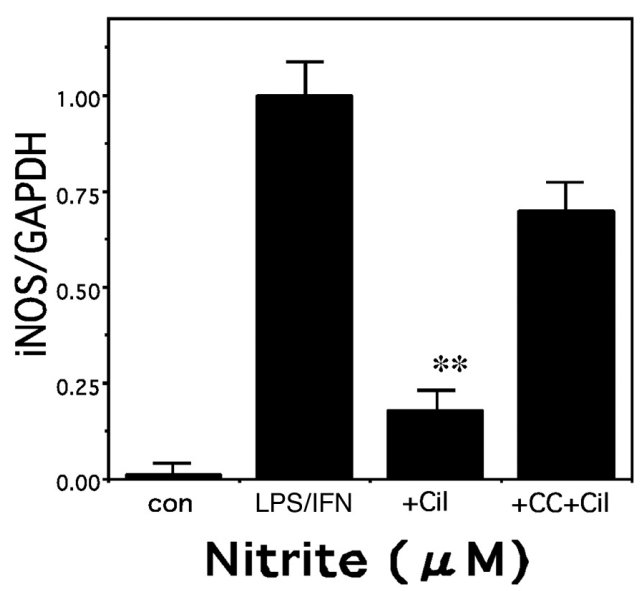

C

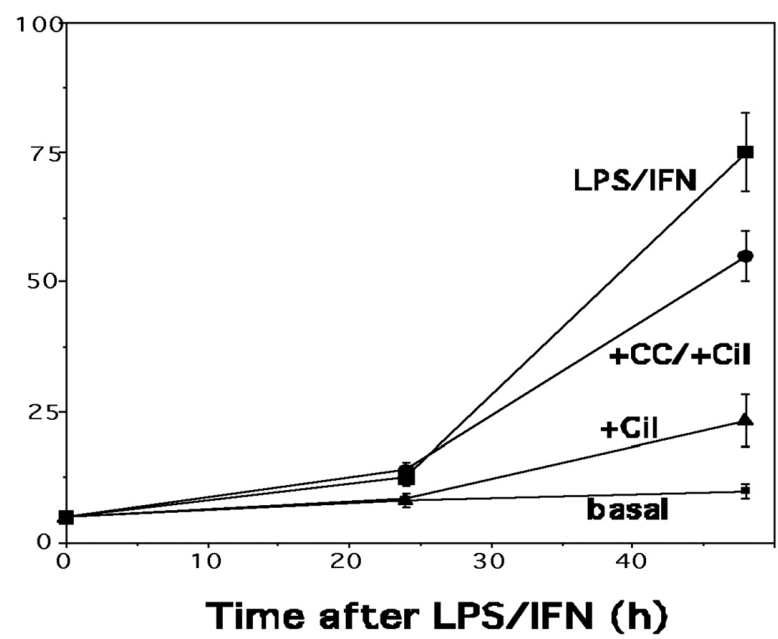

Fig. 4. (A) Effect of cilostazol $(100 \mu \mathrm{M})$ on iNOS promoter/SEAP reporter expression in stably-transfected rat VSMC. Cells were treated with LPS $(30 \mu \mathrm{g} / \mathrm{mL}$ ) plus interferon- $\gamma$ (IFN: $100 \mathrm{U} / \mathrm{mL}$ ) for $24 \mathrm{~h}$, after which SEAP activity was quantified in the cell culture medium. Values represent mean values \pm S.E.M. $(n=3) .{ }^{* *} p<0.01$ versus LPS/IFN alone. (B) Effect of cilostazol $(100 \mu \mathrm{M})$ on the LPS/IFN-induced increase in iNOS mRNA. Rat VSMC were treated with LPS/IFN for $24 \mathrm{~h}$, after which iNOS mRNA levels were quantified by real-time PCR. The results are expressed as a ratio in terms of GAPDH mRNA levels. ${ }^{* *} p<0.01$ versus LPS/IFN alone. (C) Effect of cilostazol $(100 \mu \mathrm{M})$ on nitrite production. Rat VSMC cells were treated with LPS/IFN, after which nitrite accumulation in the culture medium was measured at the indicated time points. Data represent mean values \pm S.E.M. $(n=3)$.

tion in VSMC stably transfected with an NF- $\kappa$ B reporter gene. LPS induced a 10 -fold increase in $\mathrm{NF}-\kappa \mathrm{B}$-mediated reporter gene expression, which was dose-dependently suppressed by cilostazol (Fig. 3B). We examined the effect of the AMPK inhibitor compound $\mathrm{C}$ on cilostazol-induced NF- $\kappa \mathrm{B}$ inhibition and found that it was significantly attenuated in compound C-treated cells (Fig. 3B). Similarly, it was significantly attenuated in cells transfected with siRNA for AMPK $\alpha 1$ (Fig. 3B).

\section{Cilostazol Inhibits iNOS Gene Promoter Activity, iNOS mRNA Induction, and NO Production in VSMC}

The effect of cilostazol on LPS/interferon- $y$ (IFN)-induced iNOS promoter activation in VSMC was evaluated. LPS/IFN caused potent activation of the iNOS promoter, as suggested by a $>5$-fold increase in SEAP reporter gene activity (Fig. 4A). LPS-induced iNOS promoter activity was significantly inhibited by cilostazol, which was reversed by compound $\mathrm{C}$ (Fig. 4A).

While iNOS mRNA levels approached the detection limit of real-time PCR in unstimulated VSMC, combined treatment with LPS/IFN provided a strong stimulus for iNOS mRNA expression (Fig. 4A). The observed LPS/IFN-induced increase in iNOS mRNA was substantially reduced by co-treatment with cilostazol, and this decrease was attenuated by compound C (Fig. 4B).

Nitrite accumulation, indicating $\mathrm{NO}$ synthesis, was measured in the culture medium of VSMC. Nitrite levels increased in a time-dependent manner following treatment with LPS/IFN, which was signifi- 
cantly diminished by cilostazol. This effect of cilostazol was attenuated by co-treatment with compound C (Fig.4C).

\section{Cilostazol Inhibits NF- $\kappa$ B Activation and iNOS Induction in the Aorta of LPS-Treated Rats}

We also measured p50 and p65 in nuclear extracts of aortas from LPS-injected rats untreated or treated with cilostazol. Marked increases in both p50 and p65 from very low levels were observed following LPS injection. The observed increase was significantly inhibited by cilostazol (Fig. 5A), suggesting that cilostazol suppresses LPS-induced NF- $\kappa \mathrm{B}$ activation in aortic tissue.

iNOS mRNA levels were barely detectable in the control group, while they were markedly increased in the LPS group, a finding which was largely reversed by treatment with cilostazol (Fig. 5B). Similarly, iNOS protein levels were increased in the LPS group, while low levels were observed in the LPS group treated with cilostazol (Fig. 5C).

\section{Discussion}

We recently demonstrated a potential mechanism by which cilostazol protects endothelial cells from inflammation by inhibiting the cytokine-induced expression of pro-inflammatory and adhesion molecule genes through the suppression of NF- $\kappa \mathrm{B}$ activity; this occurs via AMPK activation, not via the cyclic $\mathrm{AMP} /$ protein kinase A pathway ${ }^{2,3)}$. To confirm that this also occurs in vivo, we examined aortas from cilostazol-treated rats and found a high level of AMPK expression in the cilostazol-treated rat aorta compared with the control rat aorta, as well as strong induction of AMPK expression in the aortic smooth muscle cell layer of cilostazol-treated rats. We also observed increased AMPK protein in cultured VSMC following treatment with cilostazol. These results suggest that cilostazol may target VSMC, thereby potentiating AMPK activation in VSMC as opposed to targeting vascular endothelial cells in vivo. We thus investigated whether cilostazol might exert an anti-inflammatory effect on LPS-treated VSMC in culture and within the aortic tissue of LPS-injected rats.

We here show that cilostazol inhibits LPS-induced $\mathrm{NF}-\kappa \mathrm{B}$ activation in VSMC. We observed AMPK activation by cilostazol in VSMC and further examined whether this might be associated with inhibition of LPS-induced NF- $\kappa$ B activation. An AMPK inhibitor, compound $\mathrm{C}$, restored cilostazol-induced inhibition of NF-kB activation by LPS. It has been reported that AICAR attenuates LPS-induced activation of $\mathrm{NF}-\kappa \mathrm{B}$ via down-regulation of IkB kinase $\alpha / \beta$ activ-
A
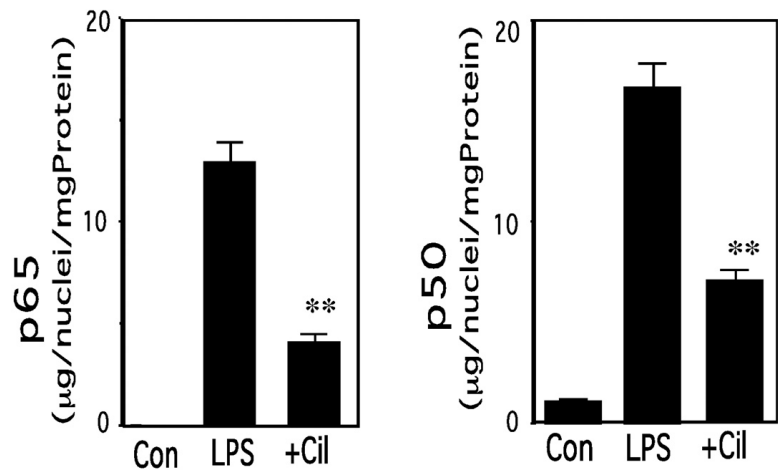

B

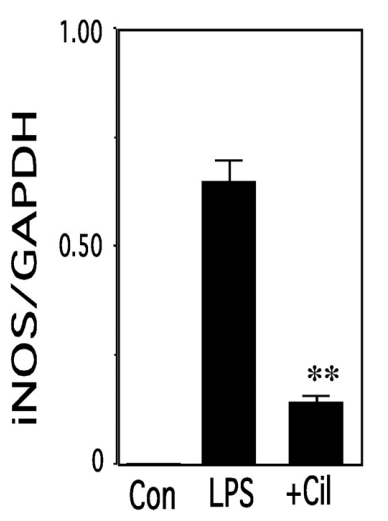

C

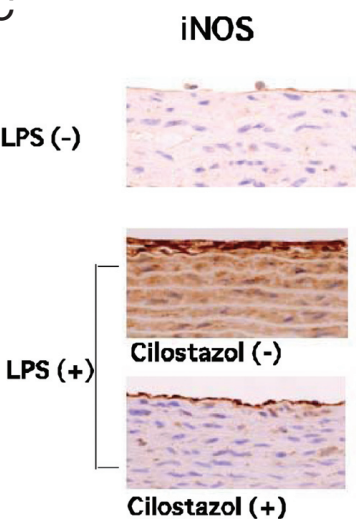

Fig. 5. (A) NF- $\kappa \mathrm{B}$ p65 or $\mathrm{p} 50$ subunits quantified within nuclear extracts from aortic tissue using a transcription factor assay kit. (B) iNOS mRNA levels measured in aortic tissue from control rats and LPS-treated rats fed cilostazol and cilostazol-free diets. (C) Immunohistochemical staining for iNOS in aortic tissue from control rats and LPS-treated rats fed cilostazol and cilostazol-free diets. Similar immunohistochemical profiles were observed in the aortic samples of 4 to 5 rats from each group. Results represent mean values \pm SEM $(n=4) .{ }^{* *} p<0.01$

ity in glial cells ${ }^{8)}$. A similar mechanism might occur in VSMC, raising the possibility that AMPK activation might inhibit cytokine-induced NF- $\kappa \mathrm{B}$ activation by suppressing IKK activity. Although an AMPK kinase assay was not performed in the present study, activation of AMPK by cilostazol is likely since the extent of AMPK phosphorylation at Thr-172 strongly reflects its activity ${ }^{9)}$, and since phosphorylation of the AMPK consensus substrate, ACC, at Ser-79 was also observed to occur ${ }^{10)}$. Among the PDE3 inhibitors, the ability to activate AMPK is specific to cilostazol, since milrinone or vesnarinone did not activate AMPK in VSMC in the present study or in endothelial cells in the previous study ${ }^{3)}$.

Cilostazol is a selective inhibitor of PDE3, by which it increases intracellular cAMP and activates 
protein kinase A (PKA), thereby inhibiting platelet aggregation and inducing peripheral vasodilation in $v_{i v o}{ }^{11,12)}$. Thus, we examined whether cAMP might be associated with cilostazol-induced activation of AMPK. We found that phosphorylation of AMPK with cilostazol was not affected by co-treatment with an adenylate cyclase inhibitor SQ 22536, and that a cell-permeable cAMP analog pCTP-cAMP did not cause AMPK phosphorylation and had no effect on cilostazolinduced phosphorylation of AMPK. Thus, AMPK activation by cilostazol occurs independently of cAMP as a protective mechanism against vascular inflammation.

We also demonstrated that cilostazol inhibits $\mathrm{NF}-\kappa \mathrm{B}$-dependent iNOS gene promoter activity, as well as the expression of iNOS mRNA, thereby inhibiting $\mathrm{NO}$ production. Inhibition of $\mathrm{NO}$ production was reversed by the AMPK inhibitor compound $\mathrm{C}$, suggesting that cilostazol inhibits $\mathrm{NO}$ synthesis by AMPK-mediated inhibition of $\mathrm{NF}-\kappa \mathrm{B}$ activation. Indeed, dietary ingestion of cilostazol significantly inhibited NF- $\kappa \mathrm{B}$ activation in the aortas of LPStreated rats. Moreover, induction of iNOS mRNA and protein expression was markedly reduced in the aortas of rats fed a diet containing cilostazol.

We gave rats a $0.1 \%$ cilostazol-containing diet, which induces serum concentrations of cilostazol at $2.0-2.5 \mu \mathrm{g} / \mathrm{mL}$ after 7 days. In humans, ingestion of two tablets of cilostazol $(100 \mathrm{mg})$ per day, the standard dosage of this drug, induces $1.0-1.2 \mu \mathrm{g} / \mathrm{mL}$ serum cilostazol concentration. Thus, this $0.1 \%$ cilostazol-containing diet is reasonable; however, experiments on cultured cells do not always represent the events at concentrations of cilostazol that occur in vivo. In this regard, further investigation is necessary, including AMPK activity measurements at various concentrations of cilostazol.

In conclusion, we demonstrated that cilostazol inhibits LPS-induced iNOS gene expression and $\mathrm{NO}$ production by blocking NF- $\kappa \mathrm{B}$ activation in VSMC. This effect of cilostazol is caused by AMPK activation through a signalling pathway independent of cyclic AMP. Although experiments on cultured cells do not necessarily represent what happens in vivo, particularly with regard to possible elevations in cAMP, our findings suggest that cilostazol-induced AMPK activation might have a beneficial effect on the vasculature in addition to its selective inhibitory action on PDE3 in patients who take cilostazol to inhibit platelet aggregation and induce peripheral vasodilation ${ }^{11-14)}$.

\section{Acknowledgements}

The authors thank Otsuka Pharmaceutical for providing the cilostazol-containing diet for rats. We are grateful to Dr. Akimoto and Miss Satoh for their technical assistance.

\section{References}

1) Goto S: Cilostazol: potential mechanism of action for antithrombotic effects accompanied by a low rate of bleeding. Atheroscler, 2005 (Suppl); 6: 3-11

2) Suzuki K, Uchida K, Nakanishi N, Hattori Y: Cilostazol activates AMP-activated protein kinase and restores endothelial function in diabetes. Am J Hypertens, 2008; 21: 451-457

3) Hattori Y, Suzuki K, Tomizawa A, Hirama N, Okayasu T, Hattori S, Satoh H, Akimoto K, Kasai K: Cilostazol inhibits cytokine-induced nuclear factor- $\{$ kappa $\}$ B activation via AMP-activated protein kinase activation in vascular endothelial cells. Cardiovasc Res, 2009; 81: 133-139

4) Camley-Cambell J, Campbell GR, Ross R: The smoth muscle in culture. Physiol Rev, 1979; 59: 1-61

5) Hattori Y, Suzuki M, Hattori S, Kasai, K: Vascular smooth muscle cell activation by glycated albumin (Amadori adducts). Hypertension, 2002; 39: 22-28

6) Hattori Y, Kasai K, Gross SS: NO suppresses while peroxynitrite sustains NF-kB: A paradigm to rationalize cytoprotective and cytotoxic actions attributed to NO. Cardiovasc Res, 2004; 63: 31-40

7) Hattori Y, Gross SS: GTP cyclohydrolase mRNA is induceds by LPS invascular smooth muscle: characterization, sequence and relationship to nitric oxide synthase. Biochem Biophys Res Commun, 1993; 195: 435-441

8) Giri S, Nath N, Smith B, Viollet B, Singh AK, Singh I: 5-aminoimidazole-4-carboxamide-1- beta-4-ribofuranoside inhibits proinflammatory response in glial cells: a possible role of AMP-activated protein kinase. J Neurosci, 2004; 24: 479-487

9) Hardie DG: The AMP-activated protein kinase pathwaynew players upstream and downstream. J Cell Sci, 2004; 117: 5479-5487

10) Dagher Z, Ruderman N, Tornheim K, Ido Y: Acute regulation of fatty acid oxidation and amp-activated protein kinase in human umbilical vein endothelial cells. Circ Res, 2001; 88: 1276-1282

11) Gotoh F, Tohgi H, Hirai S1, Terashi A, Fukuuchi Y: Cilostazol stroke prevention study: a placebo-controlled doubleblinded trial for secondary prevention of cerebral infarction. J Stroke Cerebrovasc Dis, 2000; 9: 147-157

12) Kambayashi J, Liu Y, Sun B, Shakur Y, Yoshitake M, Czerwiec F: Cilostazol as a unique anti-thrombotic agent. Curr Pharm Des, 2003; 9: 2289-2302

13) Iwamoto $T$, Kin K, Miyazaki K, Shin K, Takasaki M: Recovery of platelet function after withdrawal of cilostazol administered orally for a long period. J Atheroscler Thromb, 2003; 10: 348-354

14) Oida K, Ebata K, Kanehara H, Suzuki J, Miyamori I: Effect of cilostazol on impaired vasodilatory response of the brachial artery to ischemia in smokers. J Atheroscler Thromb, 2003; 10: 93-98 